\title{
A case of post-traumatic retroclival subdural haematoma
}

\author{
S Dayaratne ${ }^{1}$, D Rajapakse ${ }^{3}$, N Jayaweera ${ }^{2}$, S Wadanamby ${ }^{2}$, T Rajapakse ${ }^{1}$
}

Ceylon Medical Journal 2015; 60: 159-160

\section{Introduction}

Post-traumatic haematomas in the posterior fossa are uncommon, with a reported incidence of $3 \%$, and occur along the cerebellar convexity [1]. Retroclival location is uncommon and retroclival haematomas are mostly epidural. Only 6 cases of post-traumatic retroclival subdural haematomas (RCSDH) are reported in literature [2]. These are associated with significant trauma and occur in the young. Four cases of spontaneous RCSDH have also been described, three patients with coagulation disorders and one patient with pituitary apoplexy [2]. All six of the reported post traumatic RCSDH were patients aged below 20 years while the non-traumatic RCSDH were older patients. Out of the six post traumatic RCSDH, five have been managed conservatively while one required surgery due to emergence of neurological symptoms. Due to their strategic location and rarity, retroclival haematomas pose a challenge in diagnosis and management [3].

\section{Case report}

A six-year old girl was admitted with suspected head injury following a fall from a bicycle. She complained of headache. Initial neurological examination revealed a GCS score of 15 with no neurological deficit. Within the next 24 hours she developed signs of progressive bilateral upper and lower limb weakness. Computerised tomography (CT) scan of the brain revealed a hyperdense mass in the retroclival region in keeping with an extra axial haematoma. There was no fracture or atlanto-axial subluxation.

MRI scan demonstrated a subacute subdural haematoma, along the posterior aspect of the clivus, extending in to the subdural space of the upper cervical spine down to C3 level (Figure 1). Haematoma had resulted in $50 \%$ narrowing of spinal canal at craniovertebral junction and compression and oedema of the lower medulla and upper cervical spinal cord. Fat saturated sagittal sequence revealed mild oedema of the interspinous ligament between $\mathrm{C} 1$ and $\mathrm{C} 2$ suggesting flexion injury. Surgical evacuation of the haematoma was done in view of progressive neurological deterioration. Following surgery patient made a complete recovery with return of lower and upper limb power to normal
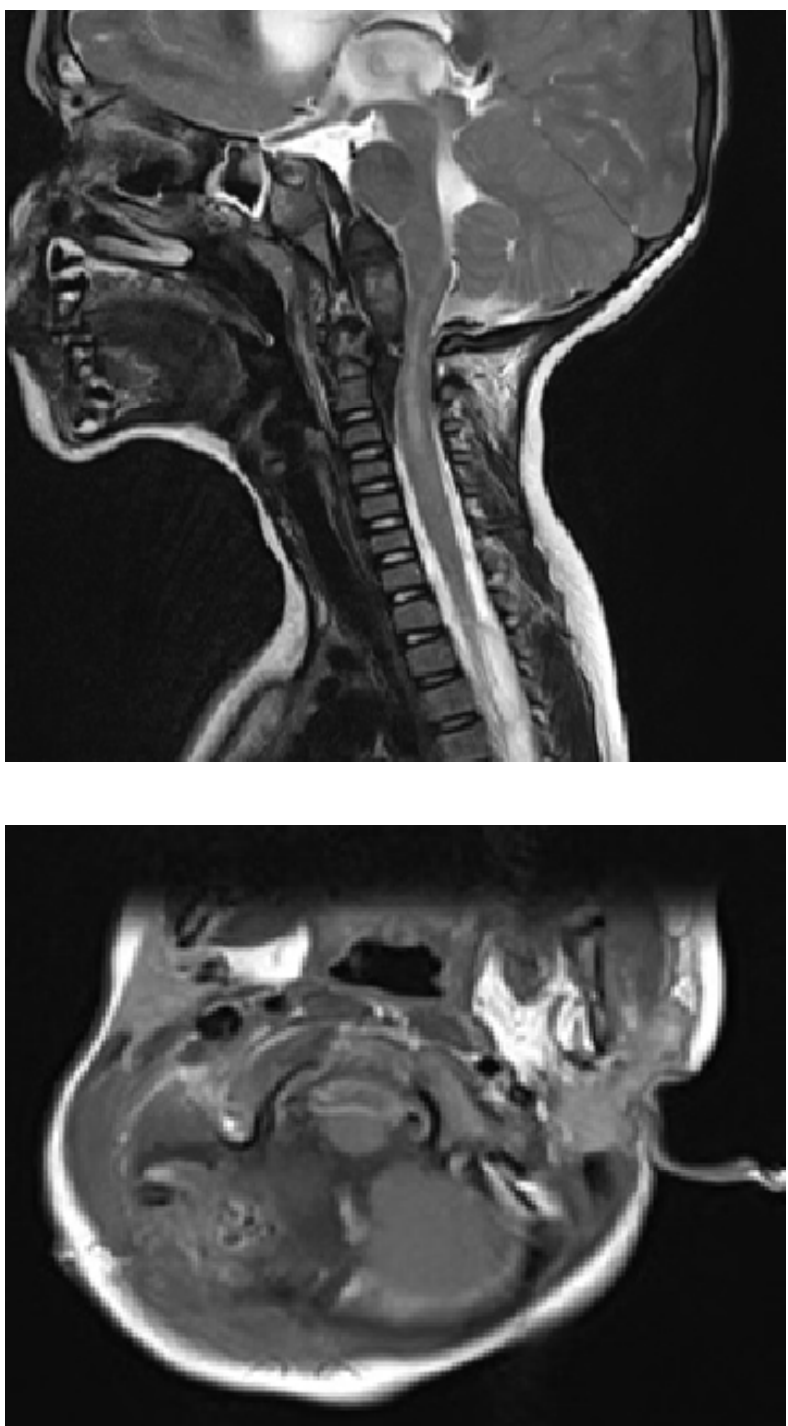

Figure 1. T2 sagittal MRI image demonstrating retroclival subdural haematoma extending down to the level of lower border of C3 vertebral body.

${ }^{1}$ Neurotrauma Imaging Unit and ${ }^{2}$ Neurosurgical Unit, National Hospital of Sri Lanka. ${ }^{3}$ Walsall Manor Hospital, UK.

Correspondence: SD, e-mail: <surangihd@gmail.com>. Received 10 July and revised version accepted 22 August 2015. 


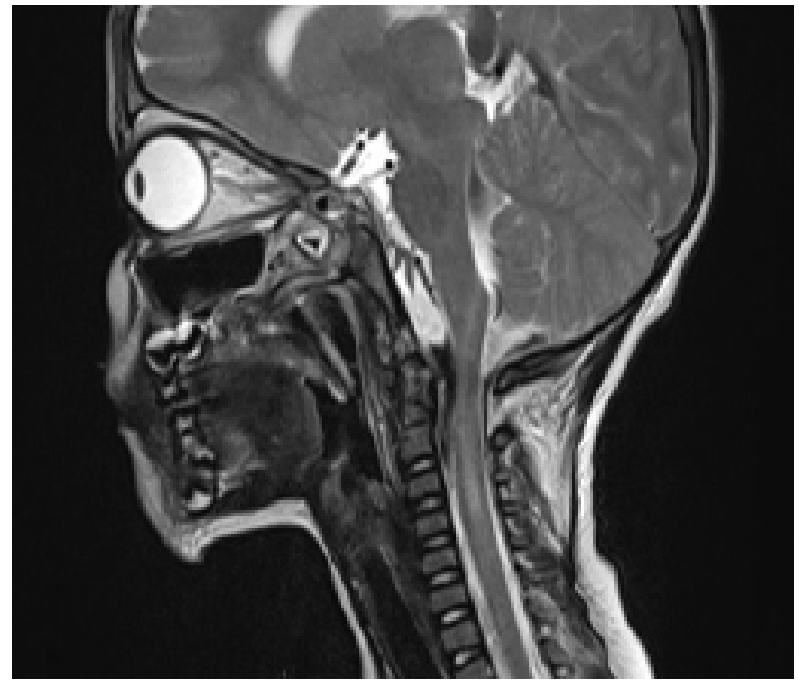

Figure 2. T2 sagittal MRI image following surgery

and GCS of 15 .

\section{Discussion}

Posterior fossa extra axial haematomas are uncommon. Post-traumatic posterior fossa haematomas are detected in approximately $3 \%$ of all CT scans taken for head injuries [1]. Differentiation between extradural (RCEDH) and subdural haematoma is important in deciding surgical approach. The main difference between a RCEDH and RCSDH is that epidural haematoma descends down to the attachment of tectorial membrane to the body of $\mathrm{C} 2$ vertebra. In comparison subdural haematoma extends below the level of $\mathrm{C} 2$. This occurs due to continuity of posterior, inner layer of clival dura with spinal dura while the anterior periosteal layer is continuous with the tectorial membrane, the upward extension of posterior longitudinal ligament $[2,4]$.

The mechanism of formation of post-traumatic $\mathrm{RCSDH}$ is not clear $[2,3]$. Bridging veins seem to be the most probable origin of RSDH [1] . In contrast, RCEDH is caused by arterial bleeding. The decision on coservative or surgical management of RCSDH is dependent on the clinical condition of the patient. The presence of blood anterior to the brainstem warrants admission to an intensive care setting to identify neurological deterioration early.

\section{Conflicts of interests}

There are no conflicts of interest.

\section{References}

1. Sridhar K, Venkateswara PG, Ramakrishnaiah S, Iyer V. Posttraumatic retroclival acute subdural hematoma: Report of two cases and review of literature. Neurol India 2010; 58: $945-8$

2. Krishnan P, Kartikueyan R, Chowdhury SR, Das S. Retroclival subdural hematoma: An uncommon site of a common pathology. Neurol India 2013; 61: 550-2

3. Ahn ES, Smith ER. Acute clival and spinal subdural hematoma with spontaneous resolution: Clinical and radiographic correlation in support of a proposed pathophysiological mechanism. J Neurosurg 2005; 103: 175-9.

4. Casey D, Chaudhary BR, Leach PA, Herwadkar A, Karabatsou K. Traumatic clival subdural hematoma in an adult. J Neurosurg 2009; 110: 1238-41. 